\begin{tabular}{|c|l|}
\hline Title & Do local properties function as cues for musical key perception? \\
\hline Author(s) & Matsunaga, Rie; A be, Jun-ichi \\
\hline Citation & $\begin{array}{l}\text { Japanese Psychological Research, 51(2), 85-95 } \\
\text { https://doi.org/10.1111j.1468-5884.2009.00391.x }\end{array}$ \\
\hline Issue Date & 2009-05 \\
\hline Doc URL & http://hdl.handle.net/2115/54929 \\
\hline Type & article \\
\hline File Information & JPR_Matsunaga.pdf \\
\hline
\end{tabular}

Instructions for use 


\title{
Do Local Properties Function as Cues for Musical Key Perception? ${ }^{1}$
}

\author{
Rie Matsunaga*2 ${ }^{2} \quad$ Jun-ichi Abe** \\ * The Japan Society for the Promotion of Science \\ (Hokkaido University of Education Sapporo) \\ ** Hokkaido University
}

Address for correspondence:

* Faculty of Education, Hokkaido University of Education Sapporo, Ainosato, Kita-ku,Sapporo,002-8501,matunaga@psych.let.hokudai.ac.jp ** Department of Psychology, Hokkaido University, N 10 W 7, Kita-ku, Sapporo, 060-0810, abe@psych.let.hokudai.ac.jp 


\begin{abstract}
A global property (i.e., pitch set) of a melody appears to serve as a primary cue for key identification. Previous studies have proposed specific local properties in a melody (e.g., the augmented fourth, the perfect fifth, etc.) that may function as further cues. However, the role of the latter in key identification is controversial. The present study was designed to investigate what kinds of local properties, if any, function as reliable cues for key identification. Listeners were asked to identify keys for 450 melodies that consisted of the same pitch set but differed in sequential constraints. Using multiple discriminant analyses, we evaluated relative contributions of as many kinds of local properties as possible (e.g., single intervals, single pitch classes in each sequential position, etc.). The results showed that, except for the pitch class of the final tone, for which interpretation should be taken cautiously, none of specific local properties examined contributed significantly to key identification. This finding suggests that, contrary to prior findings, key identification is derived from unidentified properties other than the specific local properties.
\end{abstract}

Key words: Music perception, key identification, pitch interval, pitch class. 
Key perception results from organization of a tone sequence into a hierarchical system of tonality according to a listener's internal schema that is acquired through exposure to the music of one's culture. How do listeners identify a key of a piece of music? The essential cues to key identification of any arbitrary melody have been debated and even today remained an open question.

To this issue, studies have put forward an idea of a global property of the whole melody (e.g., Abe \& Hoshino, 1990; Krumhansl, 1990; Longuet-Higgins \& Steedman, 1987). The underlying idea of a global property rests on the concept of a set of all pitch classes that constitute the complete melody, independent of their sequential ordering in the melody. Several studies provided evidence that the global property, namely a pitch set, indeed functions as a primary cue for key identification (e.g., Abe \& Hoshino, 1990; Krumhansl, 1990; Yoshino \& Abe, 2004). More specifically, these studies have demonstrated that listeners, who are familiar with Western music, identify a melody to be in a given key when the constituent pitches of this melody are all interpretable as scale tones of a certain Western-diatonic scale. For example, a tone sequence that consists of only this set of pitches: $[C, D, E, G, A, B]$, has a pitch set of six pitch classes that are consistent with four different keys: C major, G major, E minor, or A minor. Because all four keys contain the scale tones as this melody's pitch set, presumably listeners should identify the melody as belonging to one of these keys (nevertheless, it is unlikely that the choice of each of the four keys has a probability of 1/4). Thus, western-listeners perceive the key of a given melody by assimilating all constituent pitches of this melody into their 
diatonic tonal schema.

However, key perception may not be governed by a global property alone. Consider the two melodies shown in Figure 1. Although both are composed of the same set of all pitches that constitute the whole melody, they differ in the sequential arrangements of these pitches. If a global pitch set alone serves as a cue to key, then listeners should identify these two melodies to have the same key. But they do not. In fact, listeners generally interpret Melody 1 as being in $\mathrm{C}$ major and Melody 2 as being in $\mathrm{G}$ major. Thus, the idea of a global property alone does not appear to fully explain listeners' different key perception. One possible explanation for this phenomenon is that pitch set may be the only cue for key perception and perception of different keys may be derived from chance factors other than pitch set. An alternative explanation is that some properties of melodies as well as pitch set may lead to perception of the different keys.

Matsunaga and Abe (2005) examined which of the two explanations would be more appropriate. They required musically trained listeners and untrained listeners to identify a key (or a tonal center) for stimulus melodies consisting of the same pitch set but differing in the temporal arrangements of pitches. Their results suggested that, regardless of musical training, key identification of all listeners appeared to be governed not only by the global pitch set but also by certain other melodic properties.

What kinds of other melodic properties could function as essential cues in conjunction with a global property? Previous studies have proposed the idea of specific local properties. By specific local properties, we mean that one or 
several highly salient features appear within a melodic sequence, possibly at a critical sequential position, levy a strong influence on key identification. The idea of specific local properties comes from the assumption that in the field of music, listeners have acquired a practical strategy for finding the keynote within a sequence. Thus, several studies have sought to identify critical local properties upon which such strategies may be based. For example, Butler and Brown argued that one important factor in key identification is the detection of pitch intervals that are rare within a diatonic set (e.g., the augmented fourth or the diminished fifth) (e.g., Brown, Butler, \& Jones, 1994). Others have suggested that different cues function in this capacity; thus, proposed cues include the presence of the perfect fifth and either the major third or the minor third (e.g., Cuddy, 1991), an interval of minor second positioned between two adjacent tones in a tone sequence (Bharucha, 1984), the minor second in the final position (Boltz, 1989), the ascending fourth or the descending fifth in the opening position (e.g., Vos, 1999), a pitch class in the final position of a sequence (e.g., Creel \& Newport, 2002), or a pitch class in the opening position in conjunction with a pitch class in the final position (e.g., Cuddy, Cohen \& Mewhort, 1981). Finally, however, across various studies there appears to be little current consensus on this topic.

The purpose of the present study was to examine whether a specific local property might serve as an essential cue in conjunction with a global property (i.e., pitch set) for key identification of any arbitrary melody. To accomplish this, in the present research a pitch set presented to listeners was held constant, and a wide range of local properties were independently varied.

Experimentally, in order to determine that a certain specific local 
property functions as the reliable cue for musical key identification, it is necessary to demonstrate that this local property exhibits a greater influence in a key identification task than other local properties. To that end, it is important to assess the relative influences of as many different kinds of local properties as possible. Previous studies have typically limited their examinations of key identification to a few kinds of specific local properties. By contrast, the present study simultaneously evaluates relative influences of many different kinds of local properties with the aim of evaluating a range of hypotheses about local cues to key identification. In the next section, we explain our experimental rationale and MDA strategy in more detail.

Experimental rationale and MDA strategy. Our experimental strategy, and the resulting data analysis aimed at assessing key identification judgments, relies heavily upon multiple discriminant analyses (MDA; cf. Hair, Anderson, Tatham, \& Black, 1998; Klecka, 1980). In MDA of the present study, the dependent variable was assumed to reflect a few categories of key identification response (e.g., C major, G major, etc.) reported for key identification; independent variables were assumed to be each local property (treated as categorical scales, i.e., 1 for presence and 0 for absence). This technique permits determination of the relative contribution of each local property in discriminating between the categories of key identification.

In this study, all stimulus tone sequences comprise serial combinations of the same tones; thus, all six-tone sequences consisted of the same pitch set [C, $\mathrm{D}, \mathrm{E}, \mathrm{G}, \mathrm{A}, \mathrm{B}]$ but differed in the temporal arrangements of these tones. In creating different sequential arrangements, we insured that the experimental 
tone sequences contained a diversity of local properties ranging from simple local properties (e.g., the presence of perfect fifth) to complicated combinations of local properties (e.g., the perfect fifth combined with the major third in the first and third positions in a sequence). However, complicated combinations of local properties were removed as targets of examination in this study on the basis of the following principle: If a combination of local properties should prove to be salient, then it is highly likely that constituent local properties in this combination also have measureable influence. Therefore, we judged that it was appropriate to begin this undertaking by examining contributions of simple local properties to key identification.

With this caveat, the experimental tone sequences, nevertheless, offer a wide range of simple local properties. Ideally, to evaluate the relative influence of the different local properties, all local properties should be entered simultaneously (as independent variables) into a single MDA. However, this strategy was impractical in the present case due to the large number of different variables involved. Instead, we assembled numerous local properties into 10 local property groups (Figure 2):

- Pitch Class in a Particular Position group (A-1 in Figure 2)

- Pitch Interval with 0-IT in Any Position group (B-1 in Figure 2)

- Pitch Interval with 0-IT in a Particular Position group (B-2 in Figure 2)

- Pitch Interval with 1-IT in Any Position group (C-1 in Figure 2)

- Pitch Interval with 1-IT in a Particular Position group (C-2 in Figure 2)

- Pitch Interval with 2-IT in Any Position group (D-1 in Figure 2)

- Pitch Interval with 2-IT in a Particular Position group (D-1 in Figure 2) 
- Pitch Interval with 3-IT in Any Position group (E-1 in Figure 2)

- Pitch Interval with 3-IT in a Particular Position group (E-2 in Figure 2)

- Pitch Interval with 4-IT in Any Position group (F-1 in Figure 2)

The present study performed a separate MDA on each of these 10 local property groups.

All of these property groups were prepared as targets of our analyses so that meaningful analyses could be conducted. Taken together, they cover most of the specific local properties that have been proposed by the previous studies. For example, inclusion of a perfect fifth, the major third, the minor third, or the minor second can each be considered to define a single interval, regardless of a particular position. Thus, these intervals that were indicated by previous studies could be included in Pitch Interval with 0-IT in Any Position group (B-1 in Figure 2). Similarly, whether a minor second in the final position and whether ascending fourth or descending fifth in the opening position are decisive in key identifications can be determined by MDA outcomes involving the Pitch Interval with 0-IT in a Particular Position group (B-2 in Figure 2). Finally, hypotheses about a pitch class in the final position as well as proposals about the pitch class in the opening position together with a pitch class in the final position can be examined with MDA applied to the Pitch Class in a Particular Position group (A-1 in Figure 2). By doing so, we attempted to determine which, if any, of these proposed specific local properties functioned as the further cues.

Our task required listeners to directly name the key of a presented sequence. Although the key naming task much more directly assesses a listeners' perceived key than does other tasks (e.g., probe-tone task), it does require that 
listeners are capable of key naming. For this reason we employed musically trained listeners (referred to as musicians) with absolute pitch as participants; musically untrained listeners (i.e., non-musicians) cannot perform this task. In addition, musicians are typically less influenced by extraneous factors and bias. Note, however, that these differences between musicians and non-musicians does not imply that only musicians can respond to specialized perceptual cues. Previous studies indicate that musicians and non-musicians basically identify the same tonal center (e.g., Matsunaga \& Abe, 2005). Such finding suggests that perceptual cues for key identification are common to all listeners that share a common cultural exposure to music, regardless of their musical training and absolute pitch abilities.

\section{Method}

Participants. The participants were 15 undergraduate students who were highly skilled at musical performance; all reported possession of absolute pitch and could identify the key of a tone sequence. Their mean age was 20.5 years, and they had an average of 15.3 years $(S D=1.5)$ of musical training.

Materials and apparatus. Four-hundred-fifty sequences of six pitches were used as musical stimuli. All comprised the same set of six-pitches, but they differed in the temporal arrangement of these pitches. The pitch set employed was $[C, D, E, G, A, B]$, which was prepared according to the three criteria used by Matsunaga and Abe (2005). All constituent tones of this pitch set can be interpreted as scale tones of the following four keys: C major, $\mathrm{G}$ 
major, E minor, and A minor. We sought to include as many kinds of intervals as possible within tone sequences derived from $[C, D, E, G, A, B]$, given that we restricted the pitch range of the six tones to one octave. We generated two pitch sets: [C4, D4, E4, G4, A4, B4] (hereafter referred to as "Pitch Set I") and [D4, E4, G4, A4, B4, C5] (i.e., "Pitch Set II"). There were 20 possible intervals between two pitches within the two pitch sets: $( \pm 1)^{3},( \pm 2),( \pm 3),( \pm 4),( \pm 5),( \pm 7)$, $( \pm 8),( \pm 9),( \pm 10)$, and $( \pm 11)$.

It should be noted that the tone sequences in the present study did not contain the tritone intervals $( \pm 6)$, which are representatives of "rare intervals" (e.g., Brown et al., 1994). This restriction was a necessary result of selecting a pitch set of the whole melody in which all the tones could be interpreted as scale tones of four keys. To use a pitch set of the whole melody containing (+6) or $(-6)$ entails a particular situation: Keys in which all tones of a pitch set containing $( \pm 6)$ are interpretable as diatonic scale tones, are necessarily limited to only two alternatives, one major key and its relative minor key. Consider, for example, a set of three pitches $\left[\mathrm{F}_{4}, \mathrm{G}_{4}, \mathrm{~B}_{4}\right]$; here, $\mathrm{F}_{4}$ and $\mathrm{B}_{4}$ constitute the intervals $( \pm 6)$. In this case, all the tones of this pitch set are only interpretable as scale tones belonging to $\mathrm{C}$ major and its relative key (i.e., A minor). Thus, this means that specific local properties, intervals $( \pm 6)$, are not independent of a global pitch set of the whole melody as cues for key perception and the relationship between them is two sides of the same coin. Therefore, we think that it is unnecessary to distinguish the intervals $( \pm 6)$ as being different from the global pitch set in terms of essential cues because the intervals $( \pm 6)$ are unable to be separated from the global pitch set. 
All possible permutations of the six different tones yield 720 sequences from each pitch set. Out of the 1440 possible permutations, we deleted all tone sequences in which the same kinds of intervals occurred two or more times $(n=720)$ because of factor coding, and then we randomly chose 450 sequences (194 from Pitch Set I and 256 from Pitch Set II).

All sequences were monophonic isochronous melodies whose tones were contiguous and did not overlap. The duration of each tone was equal (i.e., $0.6 \mathrm{~s}$ ), for a total of $3.6 \mathrm{~s}$ per tone sequence. Intensity (MIDI velocity) was constant across all tones. The timbre was that of an acoustic grand piano. The tone sequences were created as MIDI files using Roland "Cakewalk" software installed on a Windows PC.

Procedure. The participants were tested individually. Seated in front of the two speakers, they were given a response sheet listing 12 major and 12 minor key categories. In each trial, an entire tone sequence was presented three times. There were 1.2s intervals between the presentations. After the initial three presentations, participants were allowed to listen to the given tone sequence as many additional times as they requested. They were asked to select the most plausible key for the given tone sequence on the response sheet. Subsequently, they were asked to rate their subjective confidence in their key identification on a 7 -point scale $(7=$ full confidence to $1=$ poor confidence $)$. After three practice trials, the experimental trials were presented in randomized order. The participants performed the 450 experimental trials over four days. At the end of the experiment, all participants completed a questionnaire that assessed musical background and any cuing strategies they may have used in key 
identifications.

Finally, we attempted to insure that our experimental procedure did not compel participants in a particular way based on their key identification response in a prior trial. For example, to guard against response perseveration, among other possibilities, we gave participants the following instruction: "You (i.e., a participant) must consider tone sequences given in each presentation as being independent when you select a key."

\section{Results}

Key responses and confidence ratings. The distributions of key responses for the two pitch sets were highly similar, although the mean confidence rating for Pitch Set II (4.10) was significantly higher than that for the Pitch Set I (3.98), $t(14)=3.59, p<.01$. Therefore, the data were pooled across these the two pitch sets.

As expected, key responses for all the tone sequences were limited to four of the 24 possible key response categories: C major, G major, A minor, and E minor. C major responses constituted the largest proportion of all key responses (47\%) followed by G major (29\%), A minor (13\%), and E minor (9\%). Remaining key responses were very infrequent (2\%).

Of the 450 sequences, 246 elicited response agreement among more than eight of the 15 participants for one specific key: 185 sequences for $\mathrm{C}$ major, 52 sequences for $\mathrm{G}$ major, and 9 sequences for $\mathrm{A}$ minor. There were no sequences associated with E minor. This result suggests that the participants did 
not haphazardly select one of the four possible keys for a tone sequence (given the pitch set), but instead they systematically identified one specific key by relying upon some unidentified properties other than the pitch set of the whole six-tone sequence.

Multiple discriminant analyses. Using MDA, we examined whether a specific local property made a significant contribution to distinctions among the $\mathrm{C}$ major, $\mathrm{G}$ major, and A minor keys. In this analysis we treated these three key identification responses as measurement categories of a dependent variable. Responses to 246 tone sequences (i.e., a total of 185 sequences for C major, 52 for $\mathrm{G}$ major, and nine for A minor) were each weighted by an individual responder's median confidence rating of the relevant key category in order to emphasize differences among the participants' responses to individual tone sequences. We judged a given specific local property as an essential further cue for any arbitrary melody, if results of MDA indicated that the local property satisfied the following two criteria: (1) the property was associated with one key response (e.g., C major) but not with others (e.g., G major and A minor); and (2) the property was transpositionally equivalent with each of the key responses for different absolute pitches having the same tonal functions (e.g., tonic, dominant).

Taken together, analyses for all the property groups showed that calculated discriminant functions were significant; however, Pitch Class in a Particular Position group (A-1 in Figure 2) alone revealed a certain specific local property fulfilled the above two criteria (to identify as additional cues for key identification). More specifically, this analysis indicated that the pitch 
class which occupied the final sequential position in a tone sequence reliably determined participants' key differentiations among the C major, G major, and A minor. In this paper, we will describe the detailed results for the Pitch Class in a Particular Position group, and will not describe those of other nine local groups that could not explain key responses (see Appendix).

Appendix

Figure 3 presents a detailed description of results for the Pitch Class in a Particular Position group. The dependent variable of this analysis was the key identification response with three categories ( $\mathrm{C}$ major, $\mathrm{G}$ major, and $\mathrm{A}$ minor), while independent variables were 36 properties belonging to this local group. Two discriminant functions were statistically significant, Wilks' $\lambda$ $=.241, \chi^{2}=1400.02, p<.001$ for Function 1 through 2 ; Wilks $\lambda=.696, \chi^{2}$ $=356.72, p<.001$ for Function 2. Accordingly, the following discussion is based on these two significant discriminant functions.

Figure 3(a) displays each tone sequence for each of the three key identification categories in a two-dimensional space defined by the two discriminant functions. Stars denote "centroids", which represent the most typical position for each of the three categories of key response. On the basis of locations of centroids in this space, we can infer which functions were the main source of differences among keys. Function 1 mainly provided the distinction between the $\mathrm{C}$ major and the other two keys, whereas Function 2 provided the distinction between the $\mathrm{G}$ major and $\mathrm{A}$ minor. Therefore, we assigned the label "C major-like" to the positive direction of Function 1. Similarly, we assigned the labels "G major-like" and "A minor-like" to the negative and positive directions of Function 2, respectively. 
Figure 3(b) shows pitch classes in particular positions in a space defined by the two structure coefficients. If an independent variable exhibiting structure coefficient of greater than $|0.30|$ (hereafter, we represent the absolute value of $\alpha$ as $|\alpha|)$ has the same sign in the discriminant function as that of the centroid of a certain category of a dependent variable, this indicates that this independent variable contributes positively to defining this category (cf. Hair, et al., 1998; Klecka, 1980).

Visual inspection of direction representing "C major-like" in Figure 3(b) shows that $\mathrm{C} / 6$ th (i.e., $\mathrm{C}$ in the 6 th position of a sequence) has the highest structure coefficient $(|0.26|)$. Although this value is somewhat lower than the criterion of $|0.30|, \mathrm{C} / 6$ th seems to contribute to responses of $\mathrm{C}$ major. Conversely, G/6th and A/6th that have structure coefficient of over $|0.30|$ on the negative direction of Function 1 might negatively contribute to $\mathrm{C}$ major response. Second, an inspection of the direction of representing "G major-like" shows that $\mathrm{G} / 6$ th (its coefficient $=|0.64|$ ) only exceeded $|0.30|$. This result suggests that G/6th contributed to responses of G major. Finally, inspection of the direction representing "A minor-like" shows that A/6th has the highest structure coefficient $(|0.55|)$ and G/5th has the second highest coefficient $(|0.46|)$. Moreover, D/4th has a coefficient of $|0.29|$. These results suggest that $\mathrm{A} / 6$ th, G/5th, and D/4th contributed to responses of A minor.

Of the above pitch classes in a particular position that contributed to the $\mathrm{C}$ major, $\mathrm{G}$ major, and A minor, the pitch class in the 6th position (i.e., the final position) of a sequence was common to all three key responses. Specifically, the pitch class in the final tone was $\mathrm{C}$ for $\mathrm{C}$ major, $\mathrm{G}$ for $\mathrm{G}$ major, and $\mathrm{A}$ for $\mathrm{A}$ minor. 
In these cases, $\mathrm{C}, \mathrm{G}$, and $\mathrm{A}$ could be interpreted as the tonic in $\mathrm{C}$ major, $\mathrm{G}$ major, and A minor, respectively. This indicates that the three kinds of the final tones shared the same tonal function (i.e., tonic) for each key, suggesting the pitch class in the final position made a significant contribution to the participants' key distinctions.

\section{Discussion}

We collected a large number of key response data for tone sequences that consisted of the same pitch set but exhibited different arrangements. Our results revealed that $\mathrm{C}$ major responses constitute the largest proportion of all key responses (47\%) followed by G major (29\%), A minor (13\%), and E minor (9\%). As expected, participants, who were familiar with western music, tended to perceive the given pitch set as being in major keys rather than minor keys. The distribution of key responses also showed that $\mathrm{C}$ major was chosen more than $\mathrm{G}$ major even though both keys are members of the same major mode. This also holds for the relationship between A minor and E minor. These results seemed to reflect that AP possessors tend to perceive the presented pitches as the white keys of a piano keyboard, namely those scale tones typically learned first in piano training (e.g., Miyazaki, 1988). That is, our participants, the majority of whom had had piano lessons, might be exhibiting a bias for C major (or A minor) for this reason; i.e., in these keys, all of the scale tones conform to white (not black) piano keys. In any case, the four tonal keys also contain all the constituent tones of the melody as scale tones. In this particular respect, our 
findings suggest that musical key identification is strongly delimited by a global pitch set of the whole melody.

Nevertheless, the primary purpose of the present study was to explore whether a certain specific local property, in conjunction with a global pitch set, might be responsible for finer differentiations of musical key. In particular, we assessed the relative contributions of different local properties to distinction among $\mathrm{C}$ major, $\mathrm{G}$ major, and A minor keys by employing MDA separately for each of local property groups. The results of MDA showed that the pitch class in the final position alone made a significant contribution to key identification. This means that all the groups other than except the Pitch Class in a Particular Position Group (more precisely, pitch classes of tones in any position other than the final position) made insignificant contributions.

We should interpret the results of the final tone cautiously, because it rarely happens in a general listening situation that listeners are unable to perceive the key of a melody until they hear the final tone. Our participants reported in the questionnaires that the given melodies were very similar, suggesting that they had to pay attention to subtle differences among the melodies to identify keys more properly. Considering the participants' remark, "I focused on the final tone", in such situation, the similar melodies might compel the participants to attend to the final tone because the final tone can be considered as an easily distinguishable property. In any psychological experiment, participants gradually tend to utilize the specialized strategy suitable for the requested task while performing a large number of trials. For these reasons, we cannot conclude with certainty that our finding involving the 
pitch class of final tone is a general one.

Alternatively, the present results suggest that none of the specific local properties examined contributed significantly to the participants' key identification. Those local properties included a majority of those proposed by the previous studies. In spite of the fact that previous studies report effects on key identification due to several of these local properties, our examination leads to the conclusion that the impact of such properties, as essential supplements to the global property (i.e., a pitch set), is negligible.

As an alternative to the specific local properties examined here and in prior research, there are other unexamined "more complicated" sequence properties which involve a combination of local properties. However, at least in present task, the results did not indicate that at least part of the complicated local properties had positive influences. This suggests that the idea of specific local properties does not function as essential cues for listeners' key identification for melodies that consisted of the same pitch set but exhibited the different temporal arrangements.

Our findings are relevant to a long-standing debate about the kinds of properties in any arbitrary melody that function as reliable cues for key identification. One popular hypothesis is that a combination of a global pitch set with specific local properties is the primary basis for key identification. But, as we have seen, the present results indicate that any hypothesis involving a global property-plus-specific local properties fall shorts in explaining key identification.

This conclusion leads to a consideration of a new approach. We propose 
a hypothesis that builds on the idea of a set of constituent pitches within the complete melody by introducing the idea of the accumulation of sets of constituent pitches within a melody segment provided in at given point in time. Instead of the original idea of a global property (i.e., the full set of constituent tones for melody), it is possible that listeners are differentially affected by subsets of pitches as they unfold within the melody as a whole. For instance, two melodies that share the same full pitch set $[C, D, E, G, A, B]$ can differ in their respective placements of certain subsets of pitches. Thus, consider the two melodies of Figure 1: Melody 1 opens with a subset of three pitches [C, E, G] whereas Melody 2 opens with [C, D, B]. Such differences in initial placements of pitch subsets will necessarily be correlated with distinctive differences in pitch class endings as well. Presumably, the temporal arrangements of these pitch subsets will gradually lead listeners to perception of different keys. To investigate this possibility, we are currently analyzing how listeners identify a key as musical sequences unfold over time (Matsunaga \& Abe, 2007). 


\section{References}

Abe, J., \& Hoshino, E. (1990). Schema driven properties in melody cognition:

Experiments on final tone extrapolation by music experts.

Psychomusicology, 9, 161-172.

Bharucha, J. J. (1984). Anchoring effects in music: The resolution of dissonance. Cognitive Psychology, 16, 485-518.

Boltz, M. (1989). Perceiving the end: Effects of tonal relationships on melodic completion. Journal of Experimental Psychology: Human Perception and Performance, 15, 749-761.

Brown, H., Butler, D., \& Jones, M. R. (1994). Musical and temporal influences on key discovery. Music Perception, 11, 371-402.

Creel, S. C., \& Newport, E. J. (2002). Tonal profiles of artificial scales: Implications for music learning. In C. Stevens, D. Burnham, G. McPherson, E. Schubert, \& J. Renwick (Eds.), Proceedings of the 7th International Conference on Music Perception and Cognition, Sydney, Australia: Causal Productions.

Cuddy, L. L. (1991). Melodic patterns and tonal structure: Converging evidence. Psychomusicology, 10, 104-126.

Cuddy, L. L., Cohen, A. J., \& Mewhort, D. J. K. (1981). Perception of structure in short melodic sequences. Journal of Experimental Psychology: Human Perception and Performance, 7, 869-883.

Hair, J. F., Anderson, E. R., Tatham, L. R., \& Black, W. C (1998). Multivariate data analysis, 5th ed. Upper Saddle River, NJ: Prentice Hall.

Klecka, R. W. (1980). Discriminant analysis. Quantitative Applications in the 
Social Sciences Series. Thousand Oaks, CA: Sage publications. No.19.

Krumhansl, C. L. (1990). Cognitive foundations of musical pitch. New York: Oxford University Press.

Longuet-Higgins, H. C., \& Steedman, M. J. (1987). On interpreting Bach. In H.

C. Longuet-Higgins (Ed.), Mental processes: Studies in cognitive science.

London: MIT Press. (Original work published (1971).) pp. 82-104.

Matsunaga, R., \& Abe, J. (2005). Cues for key perception of a melody: Pitch set alone? Music Perception, 23, 153-164.

Matsunaga, R., \& Abe, J. (2007). Incremental process of musical key identification. In D. S. McNamara \& J. G. Trafton (Eds.), Proceedings of the 29th Annual Cognitive Science Society. Austin, TX: Cognitive Science Society. pp. 1277-1282.

Miyazaki K. (1988). Musical pitch identification by absolute pitch possessors. Perception and Psychophysics, 44, 501-512.

Vos, P. G. (1999). Key implications of ascending forth and descending fifth openings. Psychology of Music, 27, 4-18.

Yoshino, I., \& Abe, J. (2004). Cognitive modeling of key interpretation in melody perception. Japanese Psychological Research, 46, 283-297. (Received November 5, 2007; accepted January 24, 2009) 


\section{Footnotes}

1. This research was supported in part by a Grant-in-Aid for Scientific

Research (A: 15200019) from the Japan Society for the Promotion of Science to Jun-ichi Abe.

2. We thank Dr. Masahiro Mizuto (Center for Information and Multimedia Studies, Hokkaido University) for his helpful comments on the application of multiple discriminant analysis in this article. We are grateful to Dr. Bruno Hermann Repp and Dr. Mari Riess Jones for helpful comments on earlier drafts on this article.

3. Intervals were represented as positive integers for ascending intervals and as negative integers for descending intervals (one unit $=$ a semitone). $( \pm 2)$, $( \pm 3),( \pm 4),( \pm 5),( \pm 7),( \pm 9)$, and $( \pm 11)$ could be derived from Pitch Set I. $( \pm 1)$, $( \pm 2),( \pm 3),( \pm 4),( \pm 5),( \pm 7),( \pm 8),( \pm 9)$, and $( \pm 10)$ could be derived from Pitch Set II. 
Figures

Melody $1 \stackrel{0}{2} \bullet$ C major

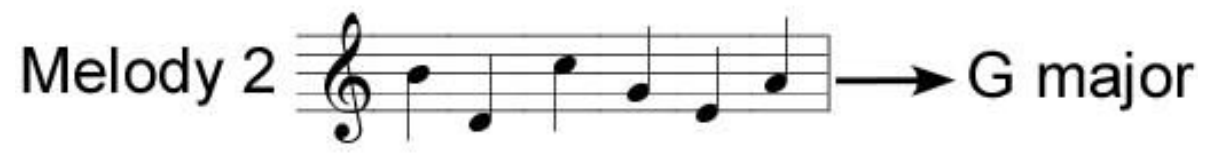




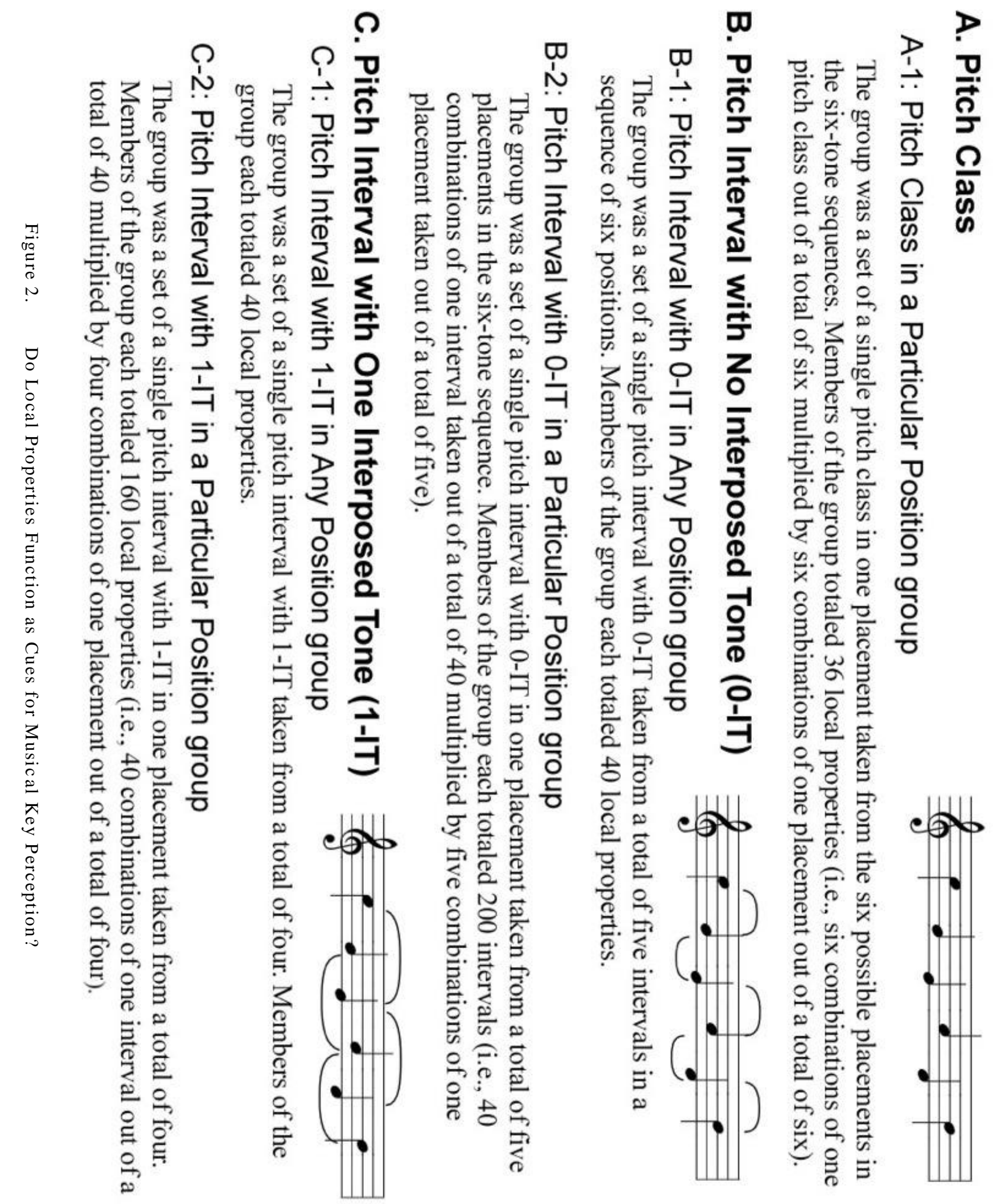




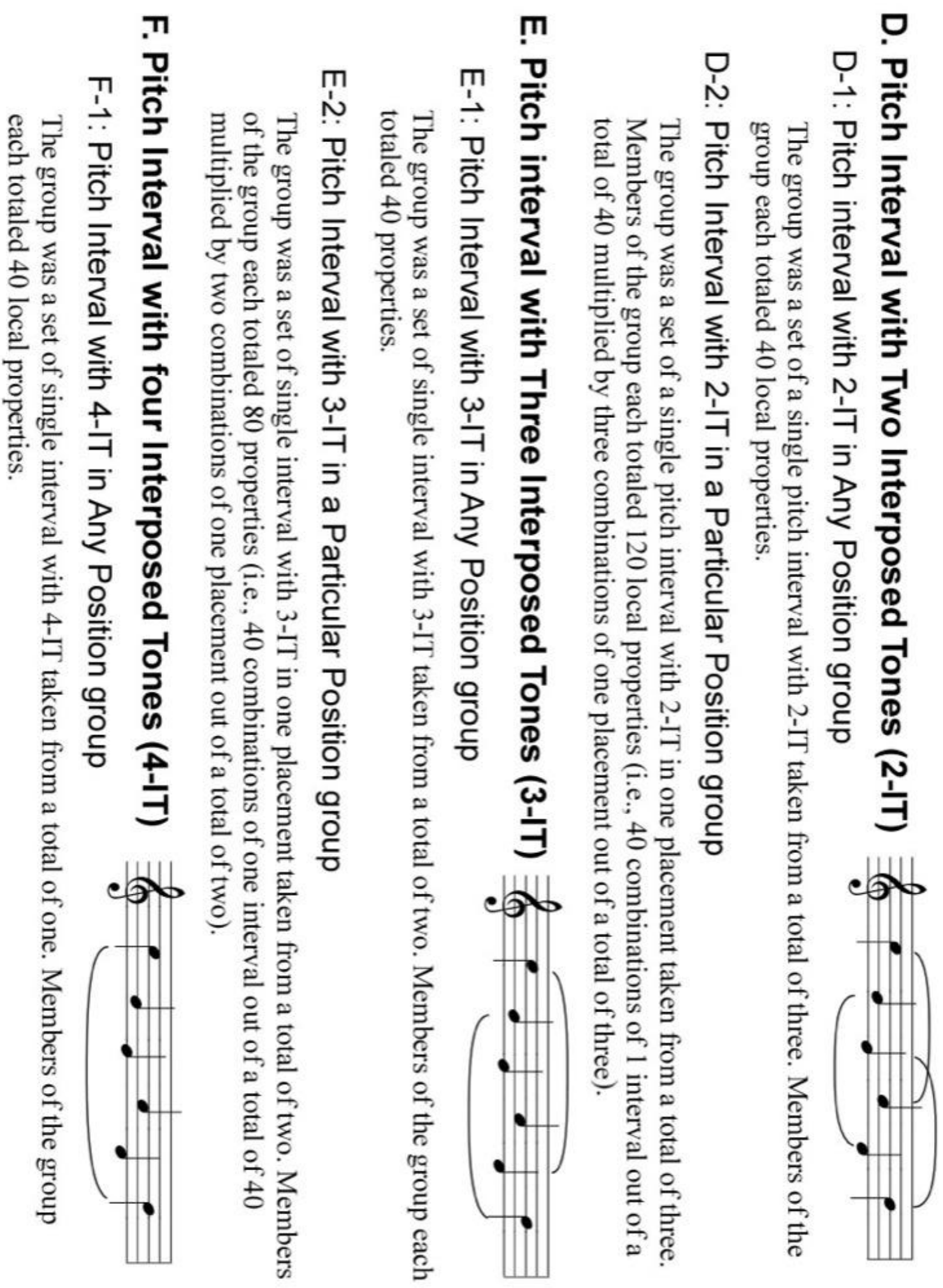




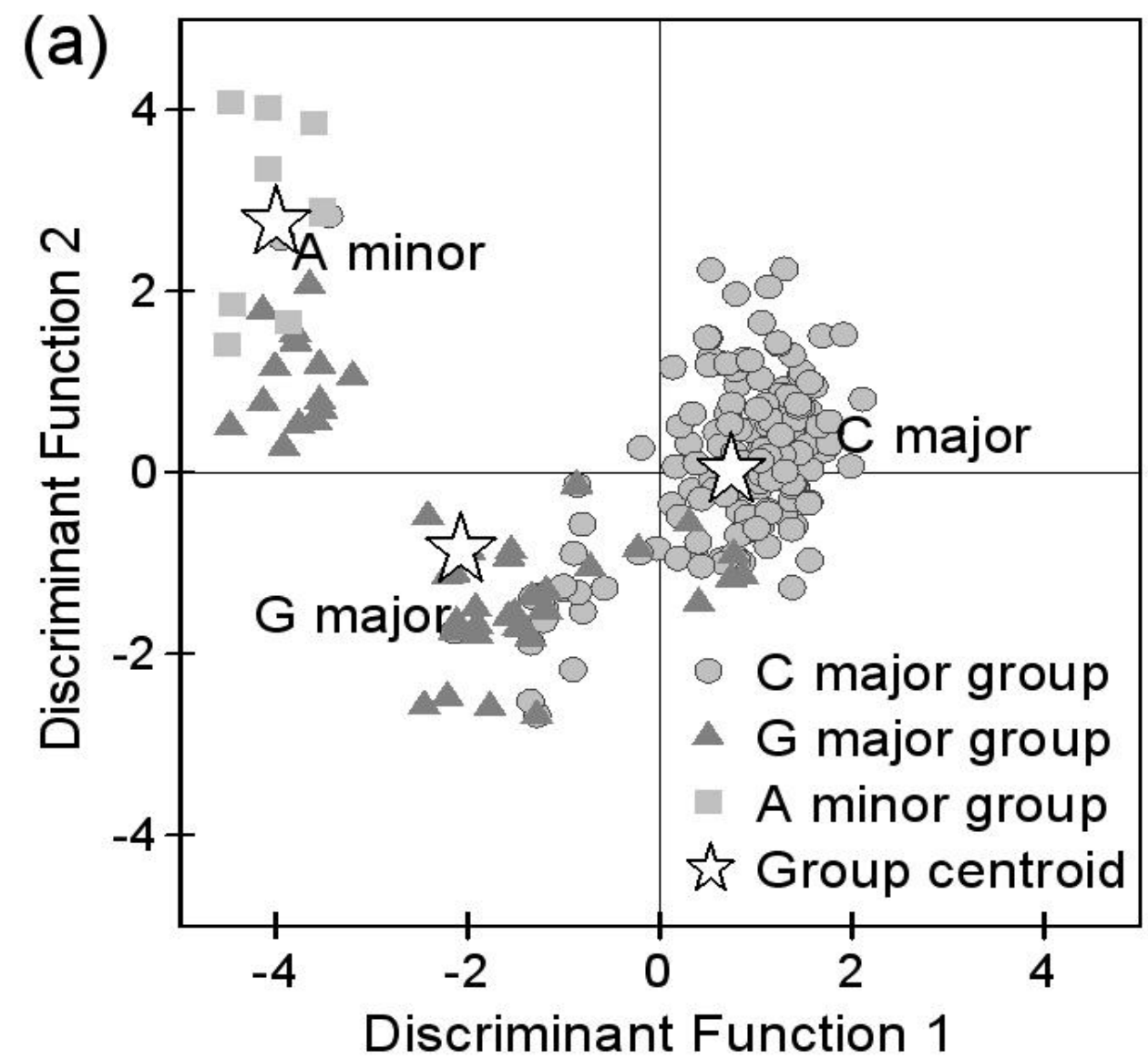

Figure 3. Do Local Properties Function as Cues for Musical Key Perception? 


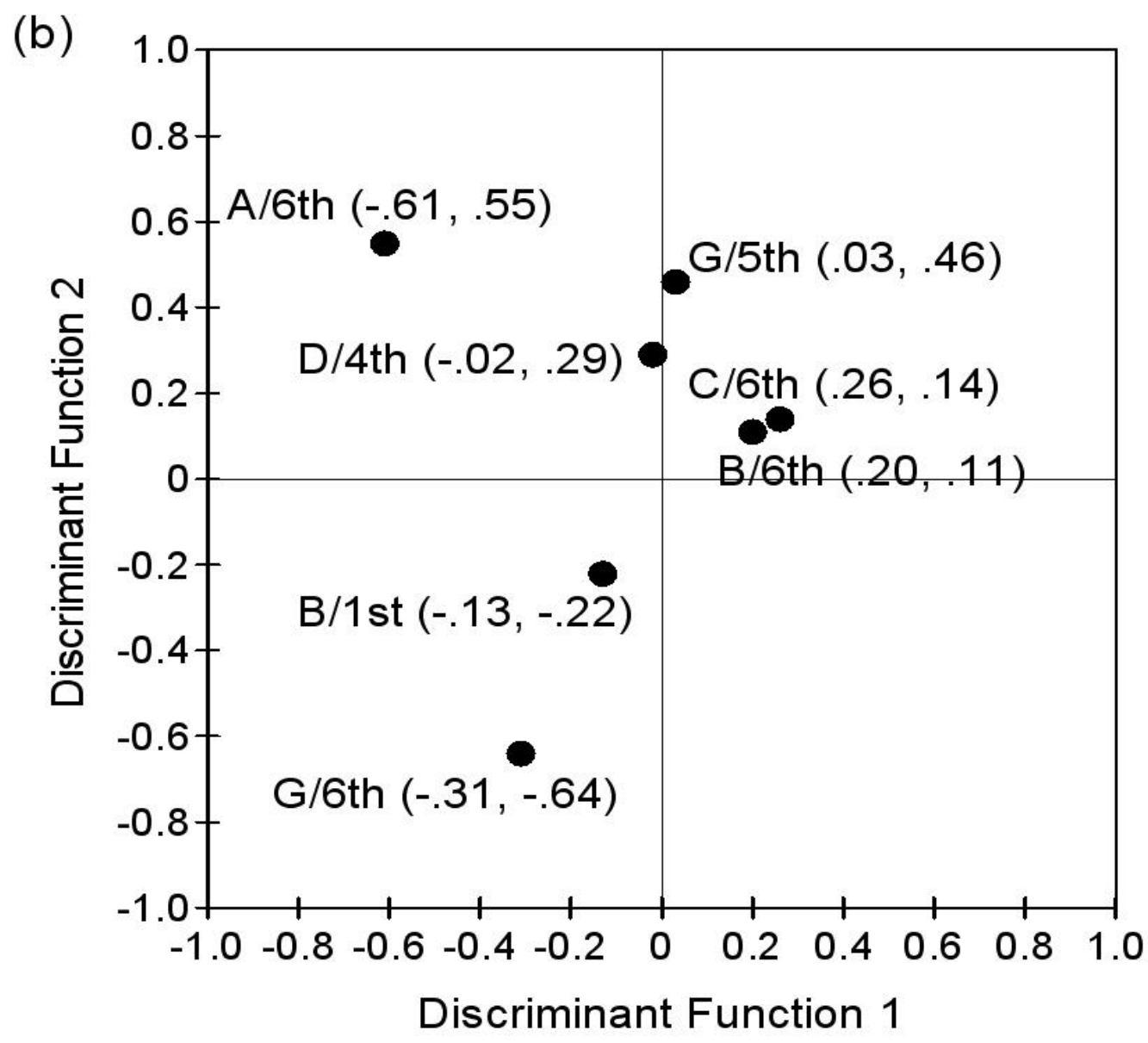

Figure 3. Do Local Properties Function as Cues for Musical Key Perception? 


\section{Figure Captions}

Figure 1. Melodies consisting of the same pitch set [C, D, E, G, A, B] differing in pitch sequence.

Figure 2. Ten local property groups as targets of our analyses.

Figure 3. Results of the analysis for the Pitch Class in a Particular Position group. Graph (a) shows tone sequences in a two-dimensional space defined by two discriminant scores. Graph (b) shows a single pitch class in a particular position in a space defined by the two structure coefficients. Pitch classes and their positions of a sequence were represented as alphabets and numbers, respectively. For example, $\mathrm{C}$ in the 1 st position of a sequence was represented as C/1st. Numbers in parentheses show structure coefficients for each function. In this paper, pitch classes with values exceeding $|0.20|$ on either Functions 1 or 2 are reported to make the graph more legible. 


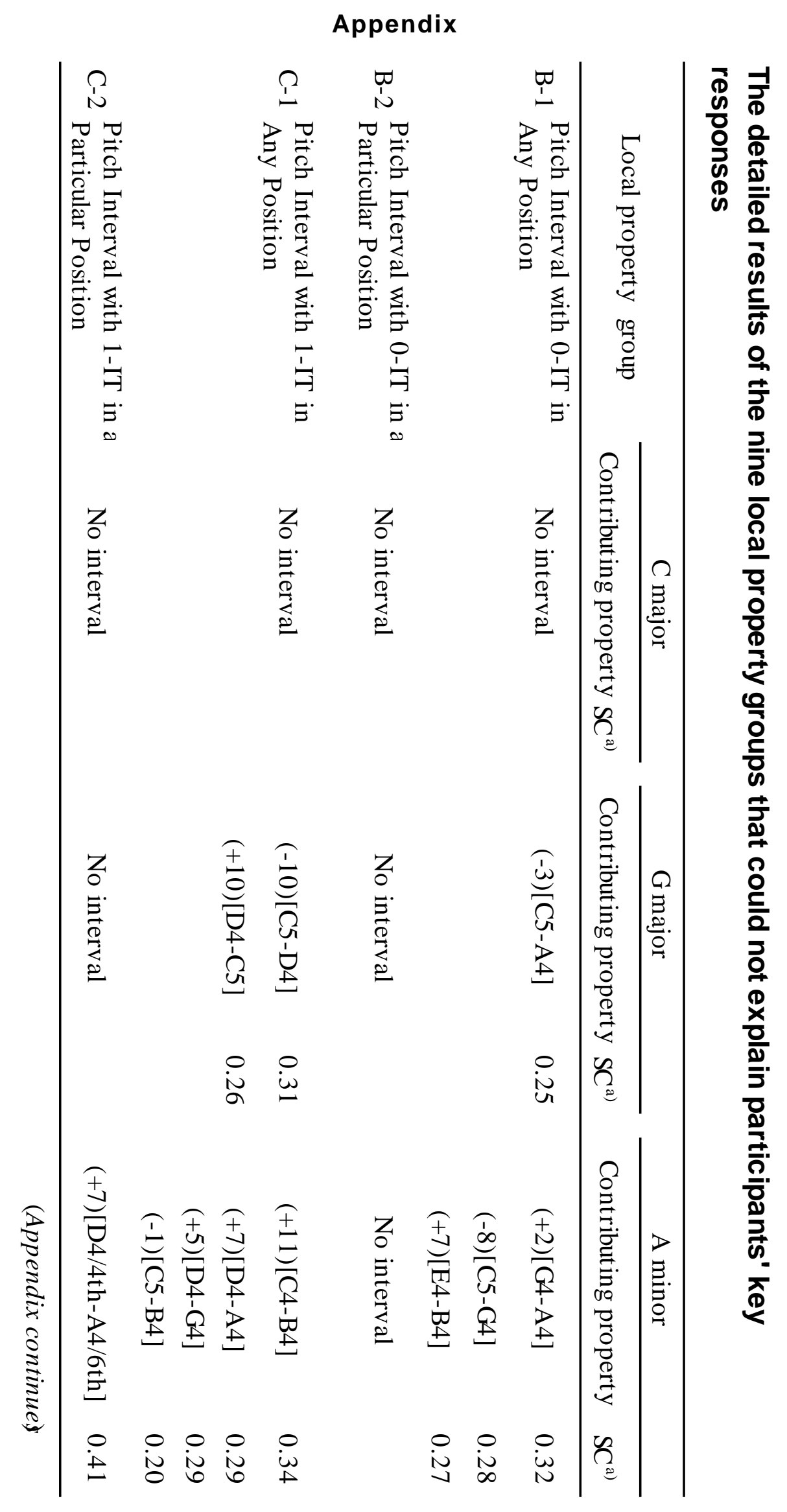




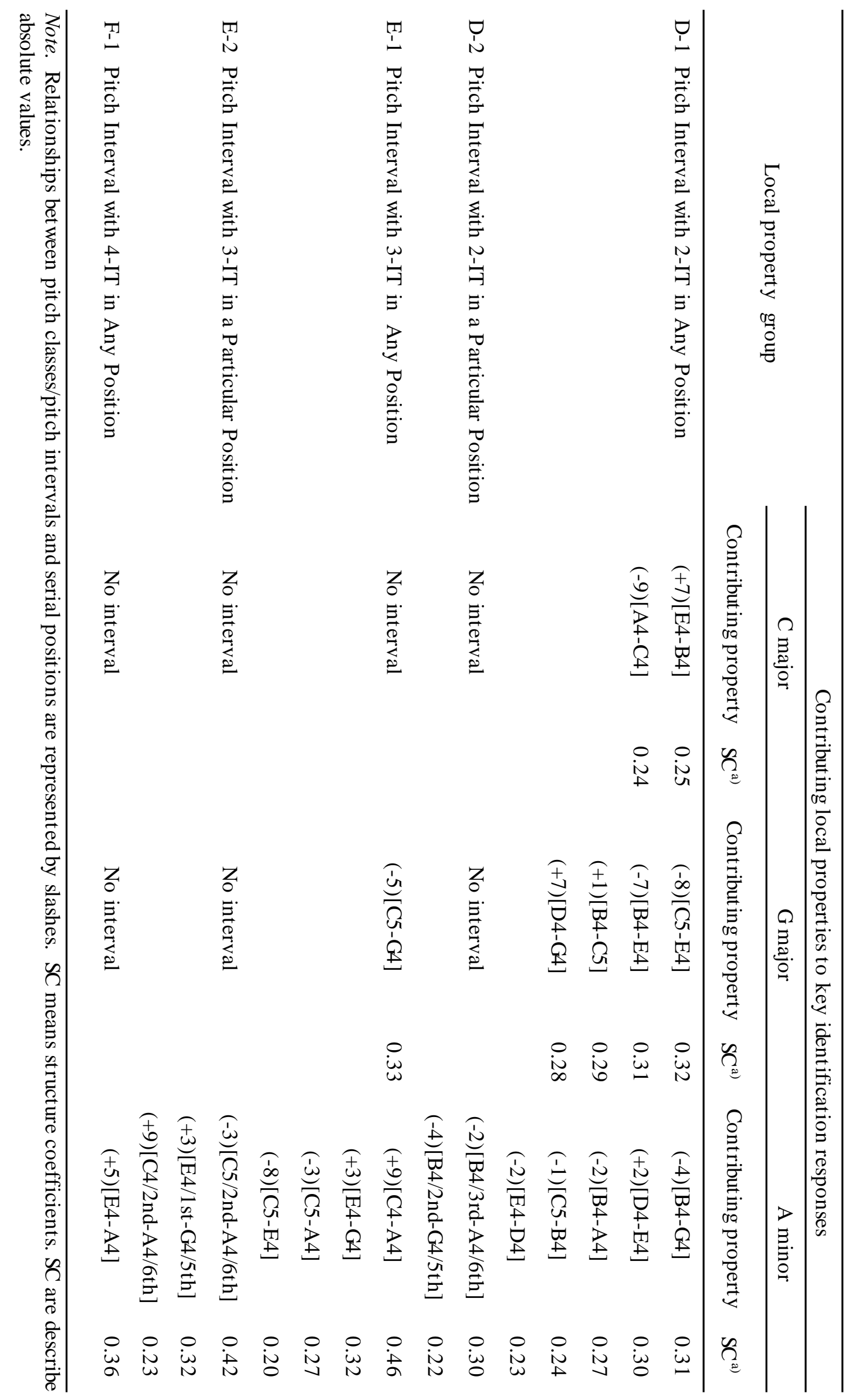

